\title{
Reine Mathematik ohne Zukunft? Eine Umfrage
}

Wir haben gefragt: Wo steht die Mathematik, zwischen Anwendungen und Nützlichkeitsdenken? Gibt es noch eine Balance zwischen Reiner und Angewandter Mathematik, und wird sich die halten? Was ist da mit Blick auf aktuelle Ausschreibungen und Stellenbesetzungen zu erwarten? Oder ist die Trennung ohnehin aufgehoben, gibt es nur noch "Angewandte Reine Mathematik" und „Rein Angewandte Mathematik"?

(MA/GMZ)

Problemlisten vom Typ „100 Jahre nach Hilbert" und die allerorten zu beobachtende Nachfrage nach Mathematik und ihren Abkömmlingen zeigen, dass wir uns um die Zunkunft der Mathematik, rein und angewandt, nicht ernsthaft zu sorgen brauchen.

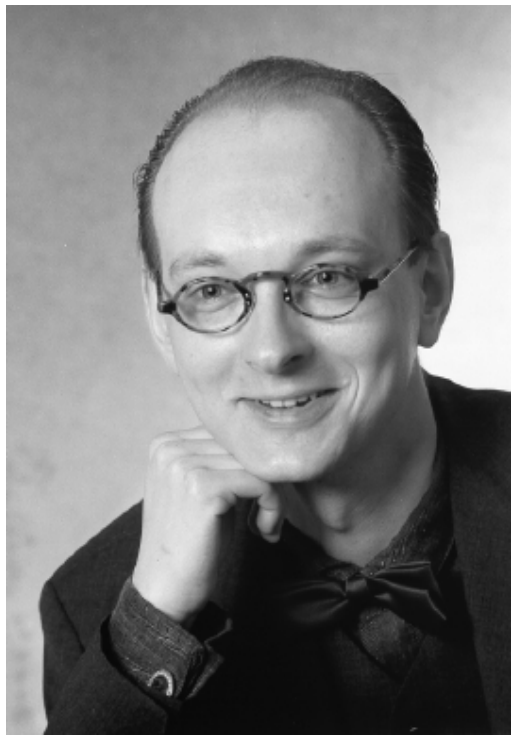

Nur stellt sich heute die Frage nach ihrer Legitimität anders als zu Beginn des 19. Jahrhunderts, als die Mathematik sich von Fremdbegründung emanzipierte, „rein“ zum Adelstitel für intellektuelle Triumpfe wurde und Mathematiker sich hierduch für Privilegien qualifizierten, die nicht nur Jacobi an Novalis' „das Leben der Götter ist Mathematik" denken ließen.

Paradoxerweise vergrößerte gerade diese "Abwendung“" in vorher ungeahntem Maße jene Anwendbarkeit der Mathematik, welche die Gewichte der gesellschaftlichen Unterstützung letztlich wieder verschob. Privilegien werden heute pragmatischer verteilt und Legitimität wird auch nicht durch den unbeholfen trotzigen Hinweis erworben, dass man schließlich nicht die Umwelt verschmutze. Statt larmoyanter Existenzangst bedürfte es eines selbstbewussten und stolzen Bekenntnisses des Grundlagenfachs zu seiner Anwendbarkeit.

Folkmar Bornemann

TU München

Alle Mathematik hat ihren Ursprung in unserer Auseinandersetzung mit der realen Welt und in der Strukturierung unserer Information darüber. Daher ist jede Mathematik „angewandt". Die Methode, einzelne Strukturelemente herauszusondern und getrennt $\mathrm{zu}$ analysieren, ist typisch für alle Bereiche der Mathematik. Daher ist jede Mathematik „rein“. Eine Gefahr sehe ich im konservativen Beharren auf Hergebrachtem und in der Beschränkung auf das Ausfeilen von Gebieten, nur weil sie schon immer betrieben wurden. Neue Impulse für die Mathematik - in allen Bereichen, (,angewandt" ebenso wie „rein") - können nur von erfrischenden neuen (einfachen) Ideen ausgehen. Solche Ideen kommen naturgemäß oft von Beziehungen zu Anwendungsgebieten.

Der Druck von Außen, direkt verwertbares Wissen und für die Unternehmen punktgenau ausgebildete Studenten zu produzieren, ist keineswegs spezifisch für die Mathematik, sondern betrifft die Grundlagenforschung in anderen Fächern noch stärker. Etwas
Überlegung zeigt, dass man ihn nicht sehr ernst nehmen kann.

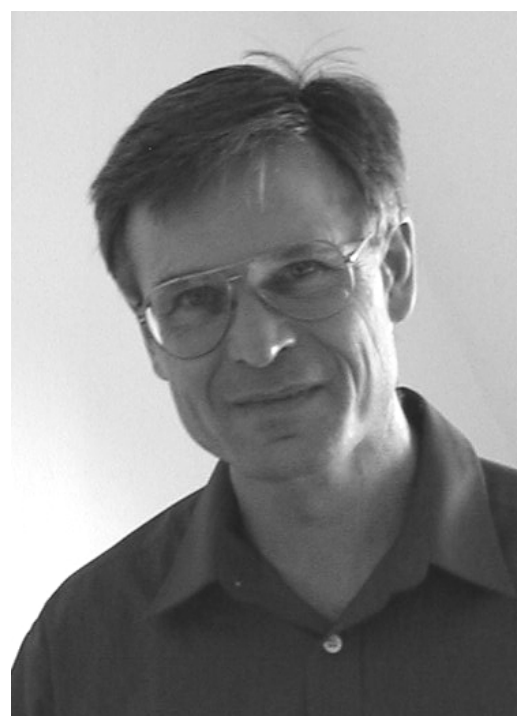

Der wesentliche Punkt ist, ob wir in der Lage sind, neue Entwicklungen flexibel aufzugreifen. Hier macht mir die Unbeweglichkeit der deutschen Universität und auch der mathematischen Fachbereiche Sorgen.

\section{Joachim Cuntz}

Münster

Das scheinbare Gegensatzpaar Reine versus Angewandte Mathematik liebe ich gar nicht: dabei klingen mir im Hintergrund $\mathrm{zu}$ sehr die Vokabeln Unreine versus Abgewandte Mathematik mit. Meine seit der Gymnasialzeit bis heute anhaltende Freude an der Mathematik speist sich aus zwei Quellen: Mathematik als Strukturdisziplin und Mathematik als algorithmische Disziplin. Beide Aspekte haben eine lange Tradition. Der Strukturaspekt wird sicher zurecht mit 
der Schönheit der Mathematik identifiziert: Schönheit muss man einfach lieben. Der algorithmische Aspekt hat in den letzten Jahrzehnten durch die Computer einen grandiosen Aufschwung genommen: dadurch ist die Mathematik zur Schlüsseldisziplin hinter den quantitativen Disziplinen geworden.

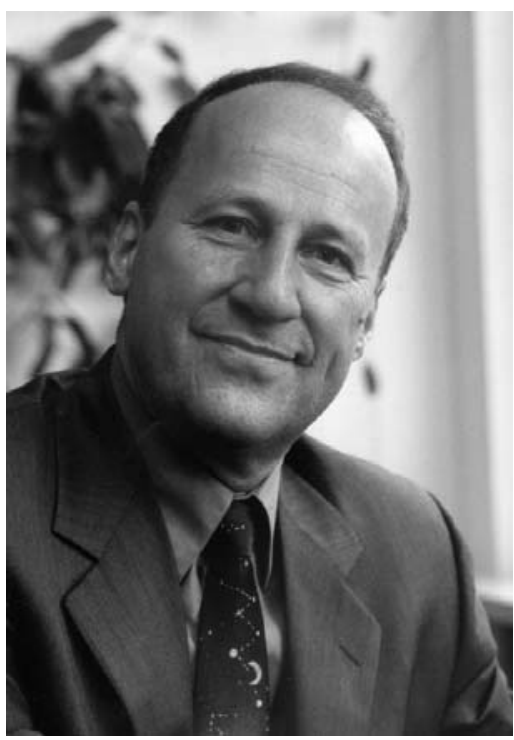

Ein weites Feld, das sich mit unglaublicher Dynamik vergrößert. Gerade das Richtige für junge Leute, die Freude an der Mathematik in all ihren Aspekten haben.

Peter Deuflhard

ZIB Berlin

Das Bestreben, subtil zwischen reiner und angewandter Mathematik zu unterscheiden, scheint in erster Linie ein innermathematisches Gesellschaftsspiel im Hinblick auf die Verteilung der Ressourcen zu sein. Wesentlich ist die Frage der Zukunft der Mathematik insgesamt.

Absolventen mit mathematischer Ausbildung sind höchst begehrt, aber immer weniger Abiturienten sind bereit, sich auf dieses Fach und seine Ansprüche einzulassen. Tun sie es, so treffen sie auf dem
Weg zum Diplom auf hohe Hürden, vor allem in den ersten Semestern und bei der Diplomarbeit. Das Programm und die Anforderungen wurden vor vielen Jahrzehnten konzipiert und seither wenig verändert. Oberstes Ziel ist und bleibt dabei von Anfang an der Fortschritt der Wissenschaft, und damit auch die Förderung des eigenen Nachwuchses. Jedoch ist die Bereitschaft der Abiturienten, die dazu nötigen Leistungen $\mathrm{zu}$ erbringen, in den letzten Jahren dramatisch abgefallen. Neue und weit weniger anspruchsvolle Studiengänge schießen in anderen Fächern wie Pilze aus dem Boden, ziehen viele Studierende an und reklamieren innerhalb der Universitäten eine Verlagerung der Ressourcen. Die Konsequenzen sind schon überall mehr oder weniger deutlich spürbar.

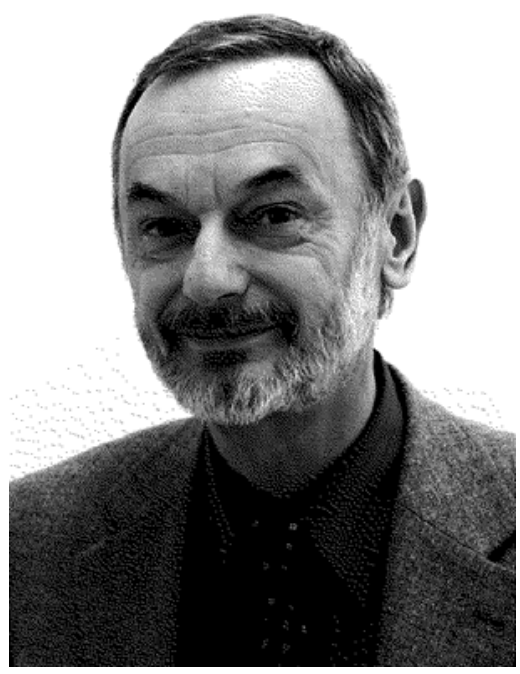

In dieser Situation sollten die mathematischen Institute ernsthaft überlegen, ob sie nicht als Alternative zum Diplom einen zweigeteilten Studiengang anbieten: Ein grundlegender Teil mit (im Vergleich zum Diplom) deutlich reduzierten Anforderungen und eigenständigem Abschluss; darauf aufbauend für besonders leistungsfähige Studierende einen zweiten Teil, der zu wissenschaftlicher Arbeit führt. Vielleicht könnten dadurch wieder mehr Abituri- enten angezogen, mehr Anfänger vor dem „Schwund" bewahrt, und so die mathematischen Institute in ihrer Existenz besser gesichert werden.

\section{Gerd Fischer \\ Düsseldorf}

Seit ein paar Jahren bemühe ich mich, die Kategorien Reine und Angewandte Mathematik nicht mehr zu verwenden, sondern nur von Mathematik zu sprechen. Dabei gehe ich davon aus, dass Mathematik wie alle anderen Wissenschaften generell nicht isoliert existiert, nicht „abgewandt" ist. Sie hat ihre Wurzeln in dem Bemühen, Wissen über die Aspekte der Welt zu schaffen, welche logisch erfasst werden können. Dabei baut sie auf elementare Erfahrungen und insbesondere Anschauungen auf. Wenn ich unscharf von Aspekten der Welt spreche, so hat das auch damit zu tun, dass es die Welt an sich nicht gibt. Es gibt nur verschiedene Vorstellungen davon, und bei der Entwicklung solcher Vorstellungen liefert die Mathematik einen kreativen Beitrag.

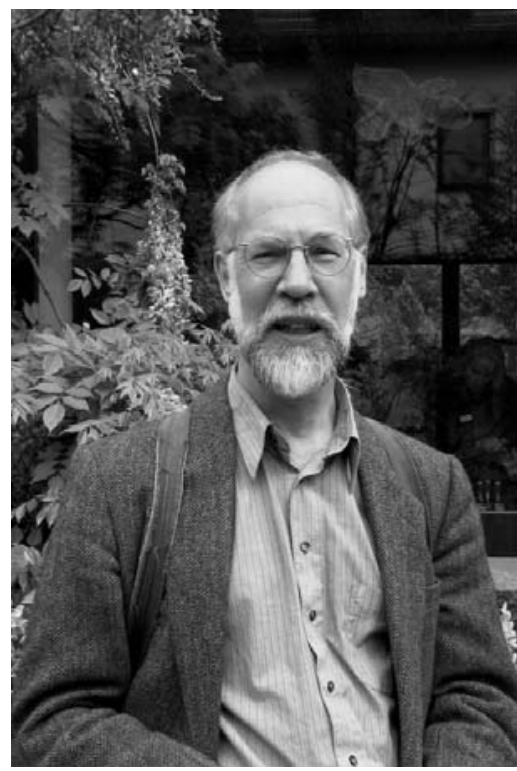

Es war immer so, dass wesentliche Fundamente der Mathematik mehr oder weniger reflektier- 
te mathematische Modellierung von aussermathematischen Sachverhalten waren. Ich verwende hier sehr bewusst das Wort Modellierung, welches heute als ein Markenzeichen angewandter Mathematik gilt. Es ist aber in Wirklichkeit ein Markenzeichen aller Mathematik. Zum Beispiel ist der Raumbegriff ein Paradebeispiel für mathematische Modellierung. Nach meiner Einschätzung ist die räumliche Vorstellung und der Versuch, sie mathematisch zu fassen, Ausgangspunkt grosser Bereiche der Mathematik. Auch Gebiete, die man gemeinhin als besonders rein bezeichnet, wie Algebra und Zahlentheorie haben einen starken Bezug zu räumlichen Vorstellungen. Für die Zahlentheorie gibt es allerdings eine unmittelbarere Begründung. Die Zahlen selbst sind in meinen Augen ein fundamentaler Bestandteil unserer Welt, der mindestens die Bedeutung der in der Physik mit einem gewaltigen Aufwand untersuchten Elementarteilchen hat.

Nun haben es komplexe mathematische Modelle und sich daraus entwickelnde Fragestellungen an sich, dass ihre Bearbeitung so schwierig ist, dass Forscher und Lerner aus den Augen verlieren können, dass sie sich - genauso wie jemand, der sozusagen im Kleinen modelliert, wenn er Verkehrsflüsse mathematisch beschreibt und $\mathrm{zu}$ optimieren versucht oder Materialien und ihre Dynamik mathematisiert - mit dem Verständnis von außerhalb der Mathematik liegenden Aspekten oder besser über die Mathematik hinausgehenden Aspekten beschäftigt. Das ändert aber nichts an der Tatsache, dass alle Mathematiker objektiv ihren spezifischen Beitrag zur Vorstellung der Welt zu leisten. Wenn wir in einer solchen Situation überhaupt das Etikett „angewandt" verwenden wollen, dann bitte für sämt- liche Mathematik - und darum sollte man lieber von Mathematik schlechthin sprechen.

Matthias Kreck

Heidelberg/Oberwolfach

I do not feel that tension between pure and applied mathematics has considerably grown recently, or that applied mathematics will make pure mathematics fade away. The relationship between the two is roughly the same as the relationship between the fundamental physics and technology, and we know very well that both have had and continue to have their own place in the history of civilization and culture. Applied mathematics is more visible thanks to computers, and much more money is invested into it, but there is nothing fundamentally new, or wrong, or threatening in this development.

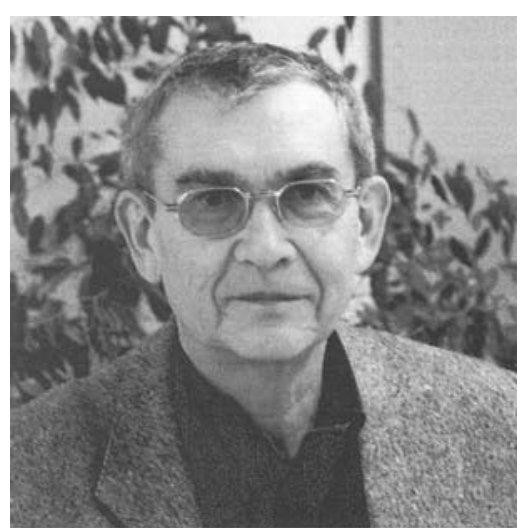

I can rather imagine that the human society will become tired or scared of consequences of unsustainable development, and that the value system of future generations will shift away from the consumer and market oriented value system of the society we live in. This can become threatening for technology in all its guises.

As for pure mathematics, there will always exist at least a handful of devotees, deeply attracted to it for no practical reason, simply because it is beautiful, and because there is something noble in this old metier, as in music and philosophy.

Yuri I. Manin

MPI Bonn

Zuerst und vor allem: Mathematik hat ungeheuer an Bedeutung gewonnen, weil sie, dank des Computers, heute in der Lage ist, Wesentliches zur Lösung praktischer Probleme aus Naturwissenschaft, Technik und Ökonomie beizutragen. Diese Bedeutung spiegelt sich darin, dass viele Vertreter dieser Fachrichtungen heute auch mathematisch arbeiten. Unser Problem als Mathematiker besteht darin, von dieser Entwicklung nicht abgekoppelt zu werden, sondern in ihr eine führende Rolle zu spielen. Da hilft keine geistige Nabelschau, sondern nur ein offener Blick nach draußen.

Schönheit oder Nützlichkeit? Warum nicht beides! Reine oder angewandte Mathematik? Sie haben unterschiedliche Motive, aber sie brauchen einander: Aus dem Theorievorrat der reinen Mathematik schöpft eine gute Modellierung, aus der Fragestellung der Praxis kommen neue Forschungsimpulse; vor allem entsteht aus der Arbeit der angewandten Mathematik die „Bedeutung" der Mathematiker für die Außenwelt, entstehen Prestige und Nachfrage nach unseren Absolventen. Deshalb bin ich gegen Trennungen der Fachbereiche in reine und angewandte Teile; es wird so eine Balance zwischen beiden innerhalb der Fachbereiche geben. Allerdings wird sich das Gleichgewicht mehr in Richtung der angewandten Mathematik verschieben: Balance meint nicht „Gleichmächtigkeit", denn sonst hätten wir in der Vergangenheit ja auch selten solche Balancen gelebt. Wir brauchen exzellente reine Mathematiker, aber dann eben nur solche - und wir brau- 
chen viele Brückenbauer, die sich in Mathematik und Praxis auskennen.

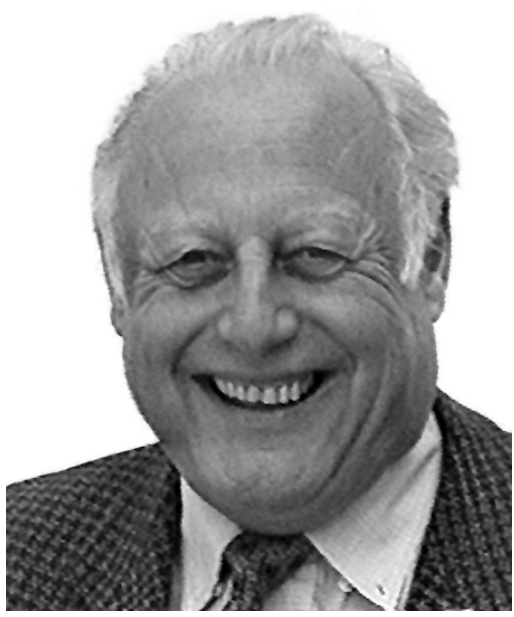

Am besten sind aber schlicht - gute Mathematiker mit echtem (!) Interesse und Blick für die Anwendungen. Reine und angewandte Mathematik sind verschieden, reine und angewandte Mathematiker können eine Personalunion eingehen. Solche „Mischwesen“ gibt es - es muss mehr davon geben und wir müssen ein Klima schaffen, in dem sie gut gedeihen. Dann hat die „Mathematik der Mathematiker" eine glänzende Zukunft!

Helmut Neunzert

Kaiserslautern

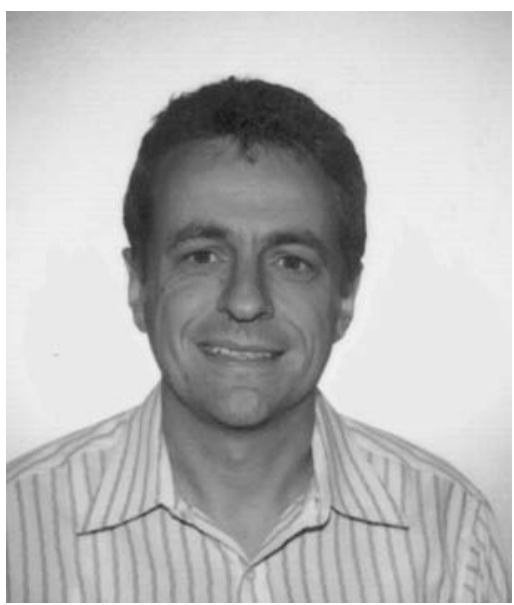

Immer mehr Gebiete der Reinen Mathematik werden in der Zukunft auf aussermathematische Problem anwendbar sein. Bereits jetzt liefern ehemals reine Bereiche wie Algebraische Geometrie oder Zahlentheorie viele neue Methoden für die Lösung praktischer Probleme. US-Firmen wie AT\&T, Microsoft, Hewlett-Packard etc. haben das klar erkannt. Angewandte Mathematik bedeutet zunehmend auch Experimentelle Mathematik und ComputerMathematik, was einen wachsenden Anteil von symbolischen (nicht-numerischen) Algorithmen beinhaltet. Diese neuen Algorithmen führen ihrerseits auf neue (tiefliegende) Probleme für die Reine Mathematik.

Ich sehe daher eine glänzende $\mathrm{Zu-}$ kunft und gute Stellenaussichten für Reine Mathematiker, solange diese bereit sind, mit offen Augen durch die Welt zu gehen.

Bernd Sturmfels

Berkeley

Seit geraumer Zeit wird von Seiten der Politik und der Öffentlichkeit ein starker Druck auf die Universitäten ausgeübt und erwartet, daß sie sich privatwirtschaftlich öffnen. Professoren sollen Unternehmen gründen, verlautet es immer öfters. Reine Grundlagenwissenschaften und damit auch die zentralen Gebiete der Mathematik geraten so immer mehr in die Defensive.

Ich denke, daß in der Tat vielerorts die reine Mathematik einen immer schwereren Stand gegenüber den angewandten Bereichen hat und die Balance verlorenzugehen droht. Es würde sich langfristig für den Fortschritt sehr ne- gativ auswirken, wenn der Mathematik ihr Fundament entzogen würde. Die Grundlagenbereiche der Mathematik im weitesten Sinne - der Begriff reine Mathematik ist mir zu eng gefaßt - sind in meinen Augen die wesentlichen Stützen für ihre ungebrochene Innovationskraft. Hieraus ziehen Anwender in unzähligen peripheren Bereichen ihren Nutzen. Die „New Technologies“ - um ein Modewort zu gebrauchen - wären ohne Logik, Algebra, Zahlentheorie, Geometrie im weitesten Sinne, Analysis, Graphentheorie und Stochstik, um nur einige zu nennen, undenkbar.

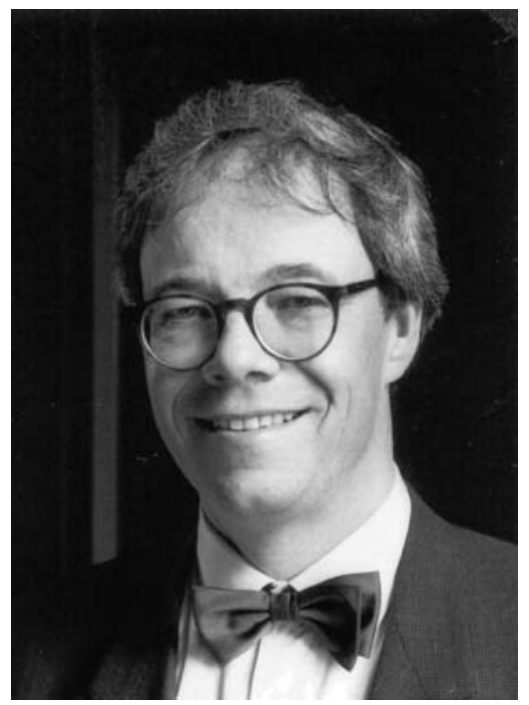

Also sollte es eher heißen: „Keine Zukunft ohne reine Mathematik!“ statt „Reine Mathematik ohne Zukunft".

Gisbert Wüstholz

ETH Zürich 